\title{
An Analysis on the Modeling Accuracy of Industrial Manipulators with Inherent Joint Elasticity
}

\author{
Rajesh Subburaman ${ }^{1}$, Mariapaola DImperio ${ }^{1}$, Jinoh Lee $^{2}$, and Ferdinando Cannella ${ }^{1}$
}

\begin{abstract}
High precision industrial applications call for equally precise functioning of industrial manipulators, which in turn requires accurate modeling of the manipulators. This paper carries out a detailed study on the modeling of industrial manipulators with elastic joints to improve their accuracy. In particular, the effect of adopting a simple harmonic drive (HD) model and ignoring a dynamic effect called low inertia coupling between the actuators and links on the model accuracy has been analyzed from a parameter estimation perspective. Since the aforementioned model characteristics have been generally ignored for high gear reduction ratios, this study is carried out with five different reduction ratios ranging from low to high, where three different models of a three-joints elastic manipulator are considered. The accuracy of the models is compared using the torque performance metrics of a predefined joint motion of the robot. Furthermore, the impact of the models with different accuracy is assessed by carrying out a state-of-the-art dynamic parameter estimation, and the resulting errors are compared to ascertain the merits of adopting a detailed elastic dynamic model of a manipulator.
\end{abstract}

Keywords - Flexible joints, Dynamic model, Modelling errors, Manipulators, Parameter estimation.

\section{INTRODUCTION}

In modern days, industrial manipulators play a vital role to achieve quality products at low cost and time. The growing demand for quality particularly increases the need for accuracy in manipulator applications. In robotic measurement applications, the accuracy requirements for manipulators are even greater, in the order of $[\mu \mathrm{m}]$. These requirements can only be met with accurate dynamic models that can facilitate precise pose estimation and control of manipulators. Lately, to reduce the overall cost of operation and for safety concerns, lightweight and elastic manipulators are mostly sought after. The inclusion of elasticity makes it even more challenging to model the non-linear dynamics of a manipulator with high accuracy and to control it effectively.

Several types of models have been used to represent the dynamics of industrial manipulators and estimate their corresponding parameters to simulate their dynamic motion. The most common one is a rigid body model, which assumes the joints and links to be rigid. This model has been used extensively in many parameters estimation works [1]-[6] to estimate the various inertial parameters-mass, center

\footnotetext{
${ }^{1}$ Authors are with the Industrial Robotics Unit, Istituto Italiano di Tecnologia (IIT), Via Morego 30, 16163, Genova, Italy (email:\{rajesh.subburaman, mariapaola.dimperio, ferdinando.cannella\}eit.it)

${ }^{2}$ Jinoh Lee is with the Institute of Robotics and Mechatronics, German Aerospace Center (DLR), 82234 Weßling, Germany (email:jinoh. leeddlr.de)
}

of mass, and inertia of each link. Interestingly, in [7], by ignoring some dynamic terms and estimating link-side torques, an elastic joint model is adapted to make use of the rigid joint model structure to estimate inertial parameters. On the other hand, citing the insufficiency of the rigid body models to accurately capture highly non-linear dynamics of manipulator systems, several different types of works have been proposed to enhance the model accuracy: in [8], the parameters are estimated offline using the rigid body models and further tuned online using a neural network to capture the dynamics which is not represented by the models. Although such online tuning approach has been fairly successful, it requires a large amount of data that encompasses different movements of the robot, which is difficult to achieve in practice; and as an alternative, online adaptive controllers have been proposed to compensate for the model inaccuracy, as reported in [9].

Since most of the robot's joints have inherent elasticity stemming from the transmission elements such as harmonic drives and belt-pulley, an elastic joint model has been considered in some parameter estimation works. In [10], a simplified elastic joint model for a single joint has been considered to estimate the inertia, joint elasticity, and actuator parameters, separately for each joint. The estimated parameters are validated with a 7 degrees-of-freedom (DoF) DLR light-weight robot by performing several point-point constant velocity movements, and the results are shown to be good. However, no quantitative data on the endeffector's accuracy has been reported. Unlike [10] where only drive flexibility has been considered, authors in [11]-[13] additionally consider flexibility in the joint due to bearings to further increase the accuracy of the elastic models. The dynamic characteristics of the model with estimated parameters are verified using frequency response functions (FRF) between motor torques (input) and its corresponding speeds (output). A close correspondence between the experimental and predicted FRF matrices along the diagonal terms has been reported in [11], [12], but the off-diagonal terms vary considerably, which suggests that the dynamic effects of distal links on a particular joint are not captured accurately by the model. Nevertheless, in [12], which also considers joint offset, misalignment, gear backlash, and kinematic parameters, the maximum absolute error of the end-effector with the estimated parameters is reported to be $0.32 \mathrm{~mm}$ over 150 different poses.

The use of elastic models in the aforementioned works is more likely to represent the robot dynamics closely when compared to those with rigid dynamic models. However, the 
reported accuracy of the former models is still insufficient for measurement applications which demand high accuracy than precision, e.g., inspection of manufactured parts. While moderately precise manipulators can be achieved with fairly accurate models and controllers, to obtain high accuracy, it is necessary to have highly accurate robot models. This calls for pushing the accuracy of elastic models even further. Interestingly and importantly, a closer look at the aforementioned elastic models reveals the following facts:

1) a simplified version of the complete elastic model ignored the low inertia coupling terms between the link and actuator dynamics; and

2) no separate model has been considered for the transmission system elements.

Both the facts are based on the assumption that the additional torque contributed by them is relatively small due to high joint reduction ratios, and doing so simplifies the model.

Accordingly, this study aims at understanding the effects of ignoring low inertia coupling terms and transmission system models on the robot's model accuracy, and thereby, propose an elastic manipulator model which can potentially improve the model's accuracy. In particular, the work makes the following contributions: 1) a detailed elastic model error analysis of ignoring low inertia coupling terms and adopting a simple HD model; 2) the aforementioned error analysis repeated across a range of transmission ratios to understand its effect and to quantify the resulting error; and 3) impact of less accurate elastic models of a manipulator on the estimation of its dynamic parameters/coefficients.

\section{PRoblem Description}

The dynamic equations of motion for an elastic joint robot in its general form can be written as given in [14] as follows:

$$
\begin{aligned}
& \left(\begin{array}{cc}
\mathbf{M}(\mathbf{q}) & \mathbf{S}(\mathbf{q}) \\
\mathbf{S}^{T}(\mathbf{q}) & \mathbf{B}
\end{array}\right)\left(\begin{array}{c}
\ddot{\mathbf{q}} \\
\ddot{\theta}
\end{array}\right)+\left(\begin{array}{c}
\mathbf{c}(\mathbf{q}, \dot{\mathbf{q}})+\mathbf{c}_{1}(\mathbf{q}, \dot{\mathbf{q}}, \dot{\boldsymbol{\theta}}) \\
\mathbf{c}_{2}(\mathbf{q}, \dot{\mathbf{q}})
\end{array}\right) \\
& +\left(\begin{array}{c}
\mathbf{g}(\mathbf{q})+\mathbf{K}(\mathbf{q}-\boldsymbol{\theta}) \\
\mathbf{K}(\boldsymbol{\theta}-\mathbf{q})
\end{array}\right)=\left(\begin{array}{c}
\mathbf{0} \\
\boldsymbol{\tau}
\end{array}\right)-\left(\begin{array}{c}
\mathbf{D}(\dot{\mathbf{q}}-\dot{\boldsymbol{\theta}}) \\
\mathbf{D}(\dot{\boldsymbol{\theta}}-\dot{\mathbf{q}})
\end{array}\right),
\end{aligned}
$$

where $\mathbf{M}, \mathbf{B}, \mathbf{c}_{i}, \mathbf{g}, \mathbf{K}$, and $\mathbf{D}$ are the link and actuator inertia matrix, Coriolis and centrifugal terms, gravity terms, joint stiffness, and damping matrices respectively. In (1), $\mathbf{q}$ and $\boldsymbol{\theta}$ are the link and motor side joint angles respectively, and $\boldsymbol{\tau}$ is the motor side torque. The link and motor side equations in (1) are coupled through the elastic torque $\tau_{J}=\mathbf{K}(\boldsymbol{\theta}-$ q) at the joints and also via $\mathbf{S}(\mathbf{q})$ the low inertia coupling matrix. In (1), two factors have often been overlooked in the conventional elastic models that can potentially affect the accuracy of those models and these are discussed below:

1) Low Inertia Coupling: $\mathbf{S}$ is usually a constant in the planar case or zero in the case of robots which has 2 links with orthogonal joint axes. In other general cases, $\mathbf{S}$ is usually ignored due to large reduction ratios (100-150), which reduces the coupling effect of $\mathbf{S}$ [14]. This simplifies the elastic model significantly but it also reduces the model's accuracy to some extent. The exact effect of ignoring $\mathbf{S}$ on the elastic model's accuracy has never been studied extensively in complex robotic systems, and this has been done in this work.

2) Simplified Harmonic Drive Model: Another important term that is being traded off for simplicity is the $\mathbf{B}$ matrix which includes the models of various transmission elements used for each robot joint. Since harmonic drives are more common in the robotics field, they are considered here. HD includes three main components: a wave generator, flex spline, and cylindrical spline. Of the three components, flex spline is flexible, and the joint elasticity is mainly due to this component. Most of the elastic joint models [11]-[13] consider the elasticity parameters but ignore the inertia of input and output components of an HD. In few other models [10], input inertia is added to the rotor inertia, and the output element is assumed to be an integral part of the link which is connected to the HD output. The insufficiencies of these simplifications have been reported elaborately in [15]-[17] with more detailed HD models. The complexity of the HD model that needs to be considered in the elastic robot model to compute a relatively more accurate $\mathrm{B}$ matrix which in turn can result in a better representation of system dynamics is also studied in this work.

For this study, we have considered three different numerical models: 1) $\bmod _{1}$ with a simple HD model, 2) $\bmod _{2}$ with a relatively complex HD model, and 3) $\bmod _{3}$ with a complex HD model and $\mathbf{S}$. A virtual model $\left(\bmod _{a d}\right)$ developed using MSC Adams software is used for comparison.

\section{System SetTings FOR AnAlysis}

A manipulator with three non-coplanar elastic joints has been considered in this work to analyse the effect of low inertia coupling and HD model. The system has 3 movable links with a reach of $1.5 \mathrm{~m}$ and weighs $139 \mathrm{~kg}$. A 3dimensional (3D) model of the system is shown in Fig. 1a. The following are the unique features and some assumptions about the system which has been considered for this study:

- The actuators of joints 1 and 2 are mounted on link 1 to reduce the inertia of the moving links by keeping them close to the robot's base.

- The motion transmission from the joint actuators to their respective links is done in two stages: belt-pulley (stage-1) and HD system (stage-2).

- Due to the above arrangement of actuators, the harmonic drive output for joint 1 is taken from the cylindrical spline. Whereas for joints 2 and 3, the output is taken from the flex splines of their respective drives.

- The joints are considered to be frictionless in order to study the other effects.

- Both joint and drive flexibility, i.e., rotational elasticity along the $X Y Z$ axes is considered for all three joints.

The aforementioned features 1-3 are common in most commercial industrial manipulators such as ABB's IRB 1600, IRB 2400, IRB 140, etc. The reason for choosing a system with 3 joints is to maximize the effect of the two factors mentioned in Section II and at the same time maintain the 
system's complexity to a moderate level. While a single joint system reduces the effects of ignoring $S$ and adopting a simple HD model, choosing a system with $>3 \mathrm{DoF}$ only increases the system's complexity. The contribution of adverse effects from joints beyond 3 is minimal due to the usage of lighter actuators and transmission systems.

\section{A. Adams Model of the System}

A virtual model of the manipulator is created using MSC Adams software along with the elasticity considered for each joint as shown in Fig. 1a. The virtual model includes the HD model but not the belt-pulley for each joint, and in the HD, the flex spline is modeled as a rigid body. These are done to keep the virtual model relatively simple and easy to use. The implementation of joint elasticity for a single joint in Adams is shown in Fig. 1b. In particular, the figure shows joint 3 connecting Link 2 and Link 3. In Fig. 1b, cylindrical spline is fixed to Link 2 and revolute joints are defined for rotor $\left(R_{\text {rotor }}\right)$, wave generator $\left(R_{W G e n}\right)$, and flex spline $\left(R_{F S p l}\right)$ keeping Link 2 as the reference. The motion transmission obtained due to the belt-pulley system is replicated by defining a coupler joint of ratio $r_{b}$ between $R_{\text {rotor }}$ and $R_{W G e n}$. Similarly, the reduced motion due to HD is modeled by defining another coupler between $R_{W G e n}$ and $R_{F S p l}$ with $r_{h d}$ set as its transmission ratio. In the above coupler joints, $R_{\text {rotor }}$ and $R_{W G e n}$ are taken as the driver joints. Since the drive compliance due to belt and HD is along the same axis $(Z)$, they are combined and only its resultant is considered here. The joint and drive compliance along $X Y Z$ axes is modeled by defining a $6 \mathrm{D}$ bushing between Link 3 and Flex Spline 3. A bushing is a connection tool in Adams that can render $6 \mathrm{D}$ compliance ( 3 translational +3 rotational) between any two rigid bodies. High stiffness and damping parameters are set for translational displacements, and for rotational compliances, the values are chosen based on the datasheets of HD and bearing systems.

\section{Manipulator Model With Joint and Drive ELASTICITY AND VARIOUS HD MODELS}

\section{A. Manipulator Model with Joint and Drive Elasticity}

The numerical model of the manipulator is developed considering the joint and drive flexibilities, the low inertia coupling between the actuators and links, and the HD model. Figure 2 shows the manipulator model with the reference frame for each joint $\left(O_{i}\right)$, joint coordinates for the input motion by each rotor $\left(\theta_{m i}\right)$, output motion after a two-stage reduction $\left(\theta_{i}\right)$, and the link motion due to drive compliance $\left(q_{i}\right)$ and joint compliance along $X\left(q_{i x}\right)$ and $Y\left(q_{i y}\right)$ axes. For the kinematic transformation between different joint reference frames, the Denavit-Hartenberg's (DH) parameters derived for the 3-joint manipulator shown in Table I are used. For instance, the representation of $O_{0}$ in terms of the ground or inertial reference frame $O_{G}$ denoted by $T_{0}^{G}$ can be computed as follows,

$$
T_{0}^{G}=R_{\theta_{0}, z} \cdot \operatorname{Tr}_{d_{0}, z} \cdot \operatorname{Tr}_{a_{0}, x} \cdot R_{\alpha_{0}, x},
$$

where $R_{\theta_{0}, z}$ and $R_{\alpha_{0}, x}$ are $4 \times 4$ homogeneous matrices with a zero displacement vector and their rotational components
TABLE I

DenaVit-Hartenberg PARAMETERs of A 3-JoInt MANiPUlator

\begin{tabular}{lllll}
\hline \hline DH Frame & $a_{i}$ & $d_{i}$ & $\alpha_{i}$ & $\theta_{i}$ \\
\hline$O_{0}$ & 0 & 0.285 & $0^{\circ}$ & $0^{\circ}$ \\
$O_{1}$ & 0 & 0.15 & $-90^{\circ}$ & $\theta_{1}$ \\
$O_{2}$ & -0.75 & -0.16 & $0^{\circ}$ & $\theta_{2}+90^{\circ}$ \\
$O_{3}$ & -0.75 & -0.0375 & $0^{\circ}$ & $\theta_{3}+90^{\circ}$ \\
\hline \hline
\end{tabular}

being a 3D rotational matrix along $z$ and $x$ axis respectively. Similarly, $\operatorname{Tr}_{d_{0}, z}$ and $T r_{a_{0}, x}$ are homogeneous matrices with unit rotation matrix and the displacement vectors are $\left[\begin{array}{ccc}0 & 0 & d_{0}\end{array}\right]^{T}$ and $\left[\begin{array}{ccc}0 & 0 & a_{0}\end{array}\right]^{T}$ respectively.

To represent $O_{1}$ in terms of $O_{G}$, the joint compliance of joint 1 along $X$ and $Y$ axes should also be taken into account. As a result, $T_{1}^{G}$ is computed as shown below:

$$
T_{1}^{G}=T_{0}^{G} \cdot\left(R_{q_{1 y}, y} \cdot R_{q_{1 x}, x}\right) \cdot\left(R_{\theta_{1}, z} \cdot \operatorname{Tr}_{d_{1}, z} \cdot T r_{a_{1}, x} \cdot R_{\alpha_{1}, x}\right),
$$

where the first expression (right side) is the homogeneous transformation of $O_{1}$ in terms of $O_{0}$ denoted by $A_{1}$. This is further transformed by the second expression representing joint 1 compliance along $X$ and $Y$ axes and finally transformed to the $\mathrm{G}$ frame by pre-multiplying with $T_{0}^{G}$.

The dynamic equation of the system considering both joint and drive flexibilities are computed using the well known Euler-Lagrange equation as shown below:

$$
\frac{d}{d t} \frac{\partial L}{\partial \dot{\Theta}}-\frac{\partial L}{\partial \Theta}=\mathbf{f}
$$

where, $L$ is the difference between the total kinetic and potential energy of the system, $\mathbf{f}$ is the generalized force vector, and $\Theta=\left[\begin{array}{llll}\mathbf{q}_{i x} & \mathbf{q}_{i y} & \mathbf{q}_{i} & \boldsymbol{\theta}_{i}\end{array}\right]^{T}$ is the generalized joint coordinate vector that includes the joint compliance, drive compliance, and reduced motion coordinates of all the joints. The total kinetic and potential energy of the system are computed as $K=\sum_{i=1}^{n_{l}}\left(K_{i}^{l}+K_{i}^{r}+K_{i}^{w g}+K_{i}^{f s}+K_{i}^{e}\right)$ and $P=\sum_{i=1}^{n_{l}}\left(P_{i}^{l}+P_{i}^{r}+P_{i}^{w g}+P_{i}^{f s}\right)$ respectively. In the above equation, $K_{i}^{l, r, w g, f s}$ and $P_{i}^{l, r, w g, f s}$ represent the kinetic and potential energy of $i^{\text {th }}$ link, rotor, wave generator, and flex spline respectively, $n_{l}$ is the number of links, and $K_{i}^{e}$ denotes the $i^{t h}$ joint stiffness. The computation of the above elements are explained briefly in [18].

Using (4), the total inertia matrix of the system $\mathbb{M} \in \mathbb{R}^{n \times n}$ can be computed as shown below:

$$
\mathbb{M}=\frac{\partial d}{\partial \ddot{\Theta}}\left(\frac{d}{d t} \frac{\partial L}{\partial \dot{\Theta}}\right),
$$

where $n=n_{j f}+n_{d f}+n_{a}$ is the total DoF of the system considered in the model, in which, $n_{j f}$ and $n_{d f}$ represents the joint and drive flexibilities, and the number of actuated joints is denoted by $n_{a}$. From $\mathbb{M}$ various equation of motion elements shown in (1) such as $\mathbf{M} \in \mathbb{R}^{n_{d f} \times n_{d f}}, \mathbf{S} \in$ $\mathbb{R}^{n_{d f} \times n_{a}}, \mathbf{B} \in \mathbb{R}^{n_{a} \times n_{a}}$, and $\mathbf{c}_{i} \in \mathbb{R}^{\left(n_{d f}+n_{a}\right) \times 1}$ can be extracted as explained in [14]. With the above equation of motion elements extracted from $\mathbb{M}$, the complete dynamic equation of motion given in (1) is obtained in symbolic form for a 3-joint elastic manipulator. To evaluate the accuracy of the numerically computed model $\bmod _{3}$, that includes $\mathbf{S}$ and all HD components as defined in (1), a pre-defined joint 


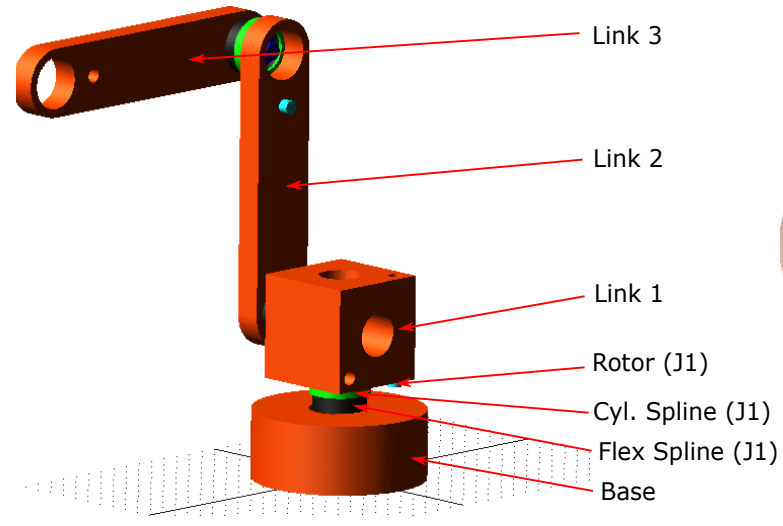

(a)

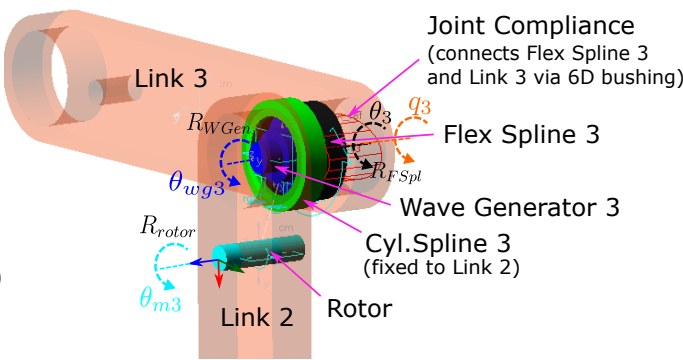

(b)

Fig. 1. (a) A virtual model of a 3 joint elastic robot modelled in Adams. (b) Implementation of joint elasticity in Adams is shown here for joint 3 along with the rotor and harmonic drive components. $\theta_{m 3}, \theta_{w g 3}, \theta_{3}$, and $q_{3}$ represents the position of rotor, wave generator, flex spline, and link respectively.

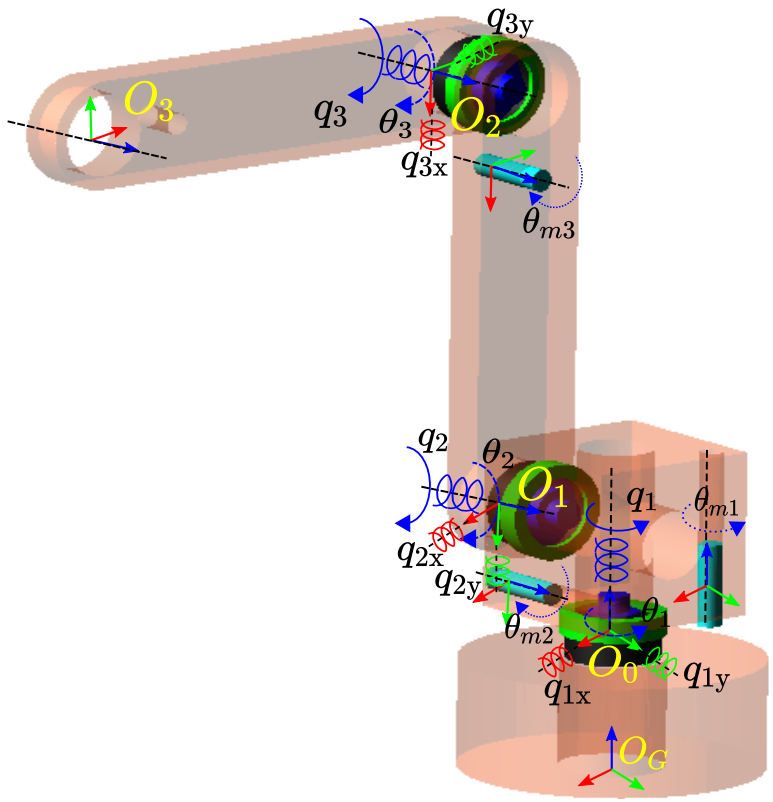

Fig. 2. A 3-joint elastic model of a manipulator is shown here with the joint reference frames, rotor motion, reduced motion after two stage reduction, link motion due to drive and joint compliance represented by $O_{i}, \theta_{m i}, \theta_{i}, q_{i}$, $q_{i x}$, and $q_{i y}$ respectively. $O_{G}$ denotes the ground or inertial reference frame of the model and the helical coils around XYZ axes show the rotational compliance along each axis.

motion is used to compute the joint $\left(\tau_{1}^{m}, \tau_{2}^{m}, \tau_{3}^{m}\right)$ and linkside torque $\left(\tau_{1}^{l}, \tau_{2}^{l}, \tau_{3}^{l}\right)$ values. These values are compared to those obtained with the virtual model $\left(\bmod _{a d}\right)$ developed using Adams (see Fig. 2), as shown in Fig. 3. For the predefined motion, a step ramp of $0.1 \mathrm{rad}$ on the output side of $\mathrm{HD}$ in $1 s$ is used. The joint side torque values here refer to those obtained at the HD output. The $r_{b}$ and $r_{h d}$ considered for this motion are 3 and 121 respectively, resulting in a total transmission ratio $\left(r_{t o t}\right)$ of 363 . This is the maximum value considered in this work to show the extreme possible errors in the numerical model. From Fig. 3, we can see that the $\tau_{i}^{m}$ of $\bmod _{3}$ correlate well to that of $\bmod _{a d}$. However, in the case of $\tau_{i}^{l}$, the torque values computed using $\bmod _{3}$ are not exactly zero when compared to those obtained using $\bmod _{a d}$.
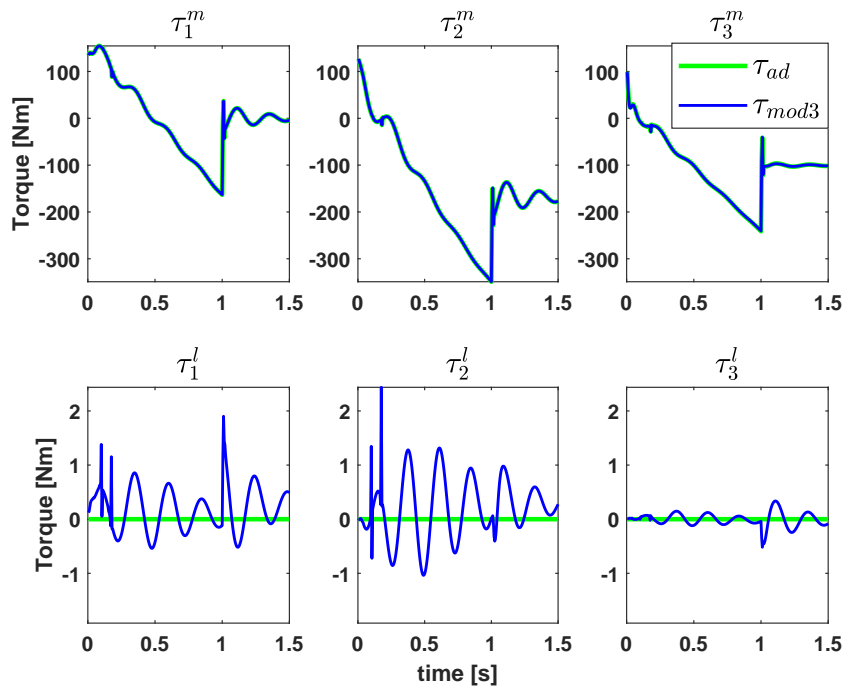

Fig. 3. Joint $\left(\tau_{1}^{m}, \tau_{2}^{m}, \tau_{3}^{m}\right)$ and link side torque $\left(\tau_{1}^{l}, \tau_{2}^{l}, \tau_{3}^{l}\right)$ values comparison made between the virtual model $\left(\bmod _{a d}\right)$ and numerically computed model $\left(\bmod _{3}\right)$ using (1), for a pre-defined joint motion.

This discrepancy is due to the 6 DoF bush joint modeled between flex spline and the link to represent the system's joint compliance in $\bmod _{a d}$, as shown in Fig. $1 \mathrm{~b}$.

\section{B. Various Harmonic Drive Models}

The conventional approach to model a HD system numerically is to consider the rotor and wave generator as a single component and the flex spline as part of the link that is being moved. Using this approach can result in erroneous torque results for the following reasons:

- The input motion from the rotor to the link is done in two stages: belt-pulley (stage-1) and HD (stage-2). This is unlike the conventional ones, where the rotor is directly connected to the HD's input component.

- Depending upon the transmission ratio of stage- 1 reduction, the dynamics of the wave generator inferred at the rotor can vary considerably.

- The dynamic properties of HD components are considerably high for bigger systems, like the one considered here, 
making it harder to ignore them.

- Finally, for applications that require high accuracy relatively more accurate HD models are required to capture its dynamics reliably.

On the other hand, there are also several complex HD models that have been reported in [16], [17], and they can capture more accurately the dynamics of systems which are harmonic driven. Though these models closely represent the harmonic drives, they are predominantly applied on 1 DoF systems Further, incorporating such complex HD models in multiDoF joint elastic systems like the one considered in this work can make it too complex for computational reasons. As a result, two different models have been considered here, to determine the one which is less complex and at the same time accurate enough to capture joint dynamics.

1) HD Model With Only Wave Generator $\left(\bmod _{1}\right)$ : The first model considers the rotor and wave generator as individual components, and the cylindrical/flex spline is taken to be an integral part of the link that is attached to HD's output. In this case, the joint compliance is assumed to be before the flex spline, and the reflected inertia at the HD output $\left(I_{h d}^{r f l}\right)$ and its resultant torque $\left(\tau_{h d}\right)$ are computed as

$$
\begin{aligned}
& I_{h d}^{r f l}=I_{w g} \cdot n_{h d}^{2}+I_{r} \cdot r_{b}^{2} \cdot r_{h d}^{2}, \\
& \tau_{h d}=\alpha_{w g} \times I_{h d}^{r f l},
\end{aligned}
$$

where $I_{w g}$ and $I_{r}$ are the inertia of the rotor and wave generator respectively, and $\alpha_{w g}$ is the acceleration of the wave generator. The above reflected inertia form appears in the diagonal actuator inertia matrix, $\mathbf{B}$, for each joint $i$ computed with their respective $I_{r}^{i}$ and $I_{w g}^{i}$ values. Since the cylindrical/flex spline components are not considered separately, the other equation of motion elements such as $\mathbf{M}$, $\mathbf{g}, \mathbf{c}$, and $\mathbf{c}_{i}$ also changes in (1). Due to the non-consideration of $\mathbf{S}$, it is taken to be a null matrix in (1) for $\bmod _{1}$.

2) HD Model With Wave Generator and Splines $\left(\bmod _{2}\right)$ : The second model is relatively more detailed when compared to the previous one, involving the splines of HD along with the rotor and wave generator components. The cylindrical/flex spline is considered to be a separate part, and it is coupled to the link through joint compliance. In this case, the reflected inertia at the HD output and its resultant torque are computed as given below

$$
\begin{aligned}
& I_{h d}^{r f l}=I_{s p l}+I_{w g} \cdot r_{h d}^{2}+I_{r} \cdot r_{b}^{2} \cdot r_{h d}^{2}, \\
& \tau_{h d}=\alpha_{s p l} \times I_{h d}^{r f l},
\end{aligned}
$$

where $I_{s p l}$ and $\alpha_{s p l}$ are the inertia and acceleration of cylindrical/flex spline depending upon the mounting arrangement of the HD. Since the cylindrical/flex spline component of HD is also considered in the model, all the equation of motion elements in (1) remain the same, except for $\mathbf{S}$, which is taken to be a null matrix since it is ignored here.

\section{Effect of Adopting a Simplified HD Model}

The $\tau_{i}^{m}$ and $\tau_{i}^{l}$ values computed for the pre-defined joint motion (Fig. 3) using three different numerical models: $\bmod _{1}, \bmod _{2}$, and $\bmod _{3}$ are compared in Fig. 4a. From the figure, we can observe a close correspondence of $\tau_{i}^{m}$ values between $\bmod _{1}, \bmod _{2}$, and $\bmod _{3}$. In the case of $\tau_{i}^{l}$, $\bmod _{1}$ and $\bmod _{2}$ differ slightly from $\bmod _{3}$ with the former showing large variation. This is attributed to the fact that the $\mathrm{S}$ term is not considered in $\bmod _{1}$ and $\bmod _{2}$. Between $\bmod _{1}$ and $\bmod _{2}$ also some difference is observed due to the different way of modeling the HD system. To quantify the differences between the models and make an efficient comparison, two performance metrics on the torque values have been considered here: maximum error $\left(e_{\max }\right)$ and root mean square error $\left(e_{r m s e}\right)$. Both $e_{\max }$ and $e_{r m s e}$ are computed with respect to $\bmod _{3}$.

To understand how the HD model error propagates as the transmission ratio is increased, five different $r_{\text {tot }}$ values $(5$, $51,128,230$, and 363) have been considered for the performance metric comparisons shown in Fig. 4b. As observed in Fig. 4a, the difference between $\bmod _{1}$ and $\bmod _{2}$ is very minimal for the $\tau_{i}^{m}$ values as $r_{t o t}$ is increased. The reason can be visualized by comparing the computation of $I_{h d}^{r f l}$ in $\bmod _{1}$ and $\bmod _{2}$, as seen in (6) and (7) respectively. The main difference is the inclusion of $I_{s p l}$ in (7) but its effect is relatively minimal since the rest of the terms are multiplied by $r_{b}^{2}$ and $r_{h d}^{2}$. The difference is relatively more pronounced in $\tau_{1}^{m}$ because HD output is at the end of the cylindrical spline instead of the flex spline, and the former's inertia is 10 times higher than the latter. This is due to a different arrangement of the HD assembly for joint 1 .

On the other hand, the difference between $\bmod _{1}$ and $\bmod _{2}$ is more pronounced on $\tau_{i}^{l}$ values and the latter reporting relatively less error. This is due to the less accurate modeling of HD in $\bmod _{1}$ since it doesn't include the spline components of HD. The modeling error appears predominantly through the $\mathbf{M}(\mathbf{q})$ and $\mathbf{c}_{\mathbf{i}}$ terms because of the absence of $I_{s p l}$ in those terms in $\bmod _{1}$, which is not the case with $\bmod _{2}$. The error in $\bmod _{1}$ and $\bmod _{2}$ and their inter differences increases linearly as $r_{\text {tot }}$ increases for joint 1 and 2 . The causality of this can be visualized in (1), in which one of the $\mathbf{c}_{\mathbf{i}}$ is a function of $\dot{\theta}$ and it is related to the rotor angular velocity $\dot{\boldsymbol{\theta}}_{m}$ as $\dot{\boldsymbol{\theta}}=r_{t o t} \cdot \dot{\boldsymbol{\theta}}_{m}$. The error observed in $\bmod _{2}$ for joint 1 and 2 is mainly due to the absence of the $\mathbf{S}$ term. In the case of joint 3 , the performance metric error remains constant for $\bmod _{1}$ as $r_{\text {tot }}$ is increased, and is 0 for $\bmod _{2}$. The former trend suggest that the error is mainly through $\mathbf{M}(\mathbf{q})$ due to the ignorance of $I_{s p l}$ because of simple HD model in $\bmod _{1}$. Since joint 3 is the last one and there are no relatively moving bodies, the components corresponding to $\tau_{3}^{l}$ in $\mathbf{c}_{\mathbf{i}}$ and $\mathbf{S}$ are 0 .

\section{EFFECT OF IGNORING LOW INERTIA COUPLING}

The effect of excluding spline components in the HD model and ignoring the $\mathbf{S}$ matrix can be analyzed by comparing $\bmod _{3}$ with the other two models $\bmod _{1}$ and $\bmod _{2}$. $\bmod _{3}$ includes the detailed HD model of $\bmod _{2}$, and it also considers $\mathbf{S}$. For the system considered here, the computed $\mathbf{S}$ matrix looks like the following:

$$
S=\left(\begin{array}{ccc}
S_{1,1} & S_{1,2} & S_{1,3} \\
0 & 0 & S_{2,3} \\
0 & 0 & 0
\end{array}\right)
$$



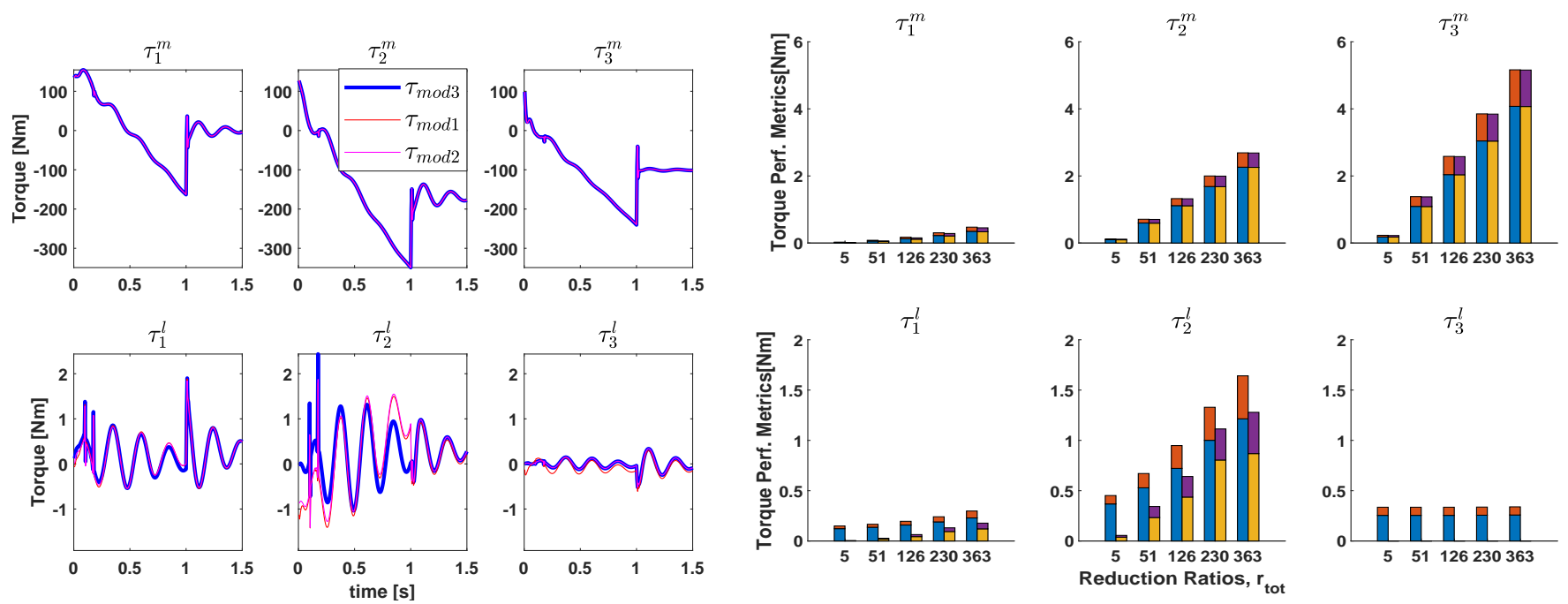

(a)

(b)
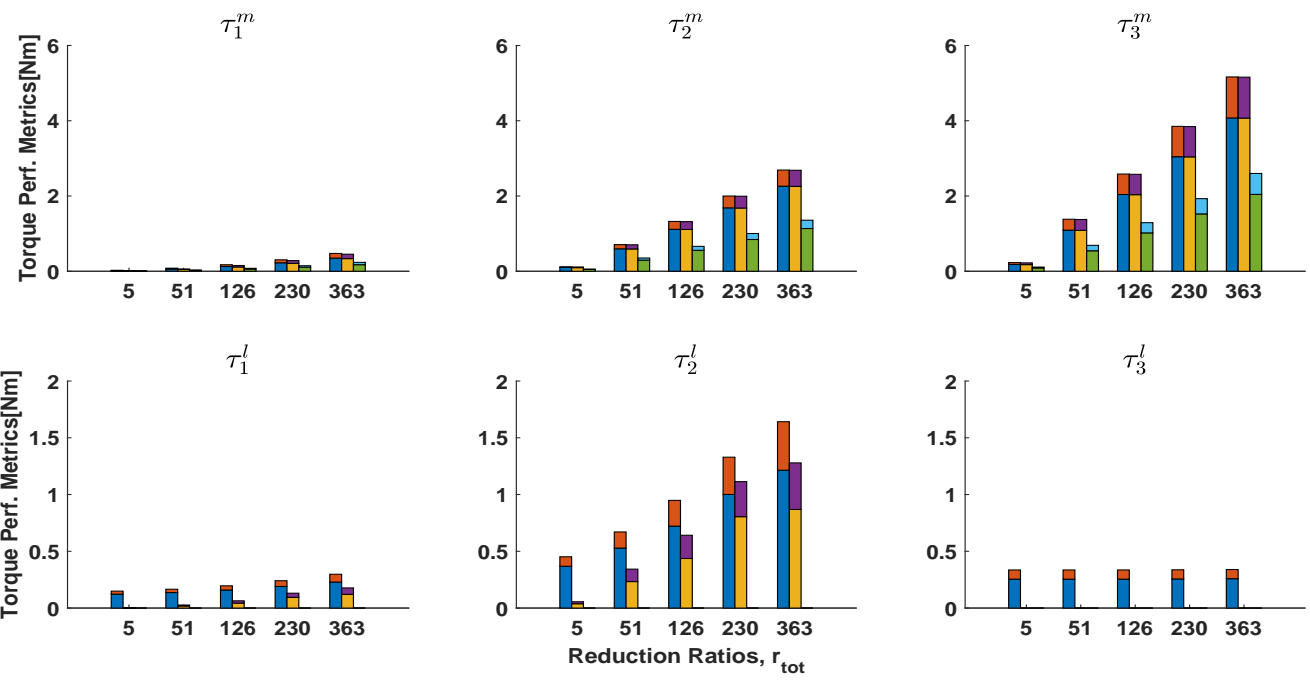

(c)

Fig. 4. (a) Joint and link side torque obtained for a pre-defined motion using three different models are shown here. The three different models considered here are: simple HD model $\left(\bmod _{1}\right)$, relatively complex HD model $\left(\bmod _{2}\right)$, and complex HD model with low inertia coupling $\left(\bmod _{3}\right)$. (b) The performance metrics (maximum error $\left(\mathrm{e}_{\max }\right)$ and root mean square error $\left(\mathrm{e}_{r m s e}\right)$ ) of the joint and link side torque values computed with different reduction ratios are compared here between $\bmod _{1}$ (1st stacked bar) and $\bmod _{2}$ (2nd stacked bar). In each stacked bar, the bottom and top bars represent $\mathrm{e}_{\max }$ and $\mathrm{e}_{\text {rmse }}$ respectively. (c) Comparison of torque performance metrics made between $\bmod _{1}, \bmod _{2}$, and $\bmod _{3}$ (3rd stacked bar).

with

$$
\begin{aligned}
& S_{1,1}=I_{z z}^{r, 1} r_{b} r_{h d} \sigma_{1}, \\
& S_{1,2}=I_{z z}^{f s, 2} \sigma_{2}+I_{z z}^{w g, 2} r_{h d} \sigma_{2}+I_{z z}^{r, 2} r_{b} r_{h d} \sigma_{2}, \\
& S_{1,3}=I_{z z}^{f s, 3} \sigma_{3}+I_{z z}^{w g, 3} r_{h d} \sigma_{3}+I_{z z}^{r, 3} r_{b} r_{h d} \sigma_{3}, \\
& S_{2,3}=I_{z z}^{f s, 3} \sigma_{4}+I_{z z}^{w g, 3} r_{h d} \sigma_{4}+I_{z z}^{r, 3} r_{b} r_{h d} \sigma_{4} .
\end{aligned}
$$

In (9), coefficients which are functions of $\mathbf{q}$ are represented in the form of $\sigma_{i}$ for brevity.

Similar to Fig. $4 \mathrm{~b}$, the performance metrics of $\tau_{i}^{m}$ and $\tau_{i}^{l}$ values are compared in Fig. $4 c$ between the three models to understand the aforementioned effect. The performance metric of $\tau_{i}^{m}$ values computed with $\bmod _{3}$ are much better than those computed using $\bmod _{1}$ and $\bmod _{2}$. Both $e_{\max }$ and $e_{\text {rmse }}$ of $\bmod _{3}$ increases linearly as the $r_{\text {tot }}$ is increased but they are $\approx 50 \%$ less when compared to those of other models. The significant improvement in the accuracy is due to the inclusion of $\mathbf{S}$ in $\bmod _{3}$ as shown in (1), and this is not the case with $\bmod _{2}$ and $\bmod _{1}$. However, torque due to the rotor and HD component's inertia, which are part of the $\mathbf{B}$ term, dominate significantly since they are multiplied by $r_{b}$ and $r_{h d}$ as shown in (7).

In the case of $\tau_{i}^{l}$, both $e_{\max }$ and $e_{\text {rmse }}$ are very minimal because of the consideration of $\mathbf{S}$ term in $\bmod _{3}$ as seen in (1). This is replaced by a null matrix $\mathbf{0}$ in the case of $\bmod _{1}$ and $\bmod _{2}$ to achieve a simplified model. Hence, the values computed with $\bmod _{3}$ are relatively more accurate than the others. Since the elements in $\mathbf{S}$ corresponding to $\tau_{3}^{l}$ are 0 as observed in (8) both $\bmod _{2}$ and $\bmod _{3}$ values of $\tau_{3}^{l}$ match exactly. The constant error observed with $\bmod _{1}$ for $\tau_{3}^{l}$ is due to the exclusion of spline component in its HD model. 


\section{ESTIMATION ERROR OF DIFFERENT MODELS}

Sections IV-B and V demonstrate the likely error to occur in the joint and link-side torque values due to different models, but the relative torque error appears to be minimal. To understand its exact impact, it is necessary to assess the effect of each model's torque errors in estimating their respective dynamic parameters $\Psi$, i.e., the mass, center of mass, inertia of different components, and the stiffness and damping of joints. Few works have already been proposed to estimate the dynamic parameters of a manipulator, such as [6], [7], [19], etc. In this work, we have adapted the estimation procedure proposed in [6] for our elastic joint model. For the estimation here, we have set $\mathbf{q}_{i x}, \mathbf{q}_{i y}$, and their respective derivatives to 0 . The elements of equation of motion shown in (1) $\mathbf{M}(\mathbf{q}), \mathbf{S}(\mathbf{q}), \mathbf{B}, \mathbf{g}(\mathbf{q}), \mathbf{K}(\mathbf{q}, \boldsymbol{\theta}), \mathbf{D}(\dot{\mathbf{q}}, \dot{\boldsymbol{\theta}})$, and $\mathbf{c}_{i}(\mathbf{q}, \dot{\mathbf{q}}, \dot{\boldsymbol{\theta}})$ are all functions of the dynamic parameters $\Psi$. Since the aforementioned elements are also functions of $\mathbf{q}$, $\dot{\mathbf{q}}, \ddot{\mathbf{q}}, \boldsymbol{\theta}, \dot{\boldsymbol{\theta}}$, and $\ddot{\boldsymbol{\theta}}$, they can be rewritten in a linear regression form as shown below

$$
\left[\begin{array}{ll}
\mathbf{0} & \tau
\end{array}\right]^{T}=\Phi(\mathbf{q}, \dot{\mathbf{q}}, \ddot{\mathbf{q}}, \boldsymbol{\theta}, \dot{\boldsymbol{\theta}}, \ddot{\boldsymbol{\theta}}) \Psi
$$

where $\Phi$ is the regression or identification matrix, and $\Psi$ is the dynamic parameter vector that needs to be estimated, and this is observed to be linear as seen in (10). $\Phi$ can be computed easily since it is a function of motion parameters.

\section{A. Dynamic Parameters and Coefficients Estimation}

To estimate the parameters reliably, it is necessary to design motions that can sufficiently excite the parameters which are to be estimated. We have used a parametric way based on the Fourier series to design trajectories, and the parameters are optimized using a pattern search algorithm from Matlab. For more details please refer to [5]. The resulting optimal excitation trajectory is used to simulate the virtual Adams model, and the corresponding joint and linkside torque values are recorded. Using the linear regression form in (10), the parameter/coefficient vector $\Psi$ can be estimated in a linear least-square form $\Psi_{l s}$ as follows:

$$
\Psi_{l s}=\left(\mathbf{F}^{T} \mathbf{F}\right)^{-1} \mathbf{F}^{T} \mathbf{b},
$$

with

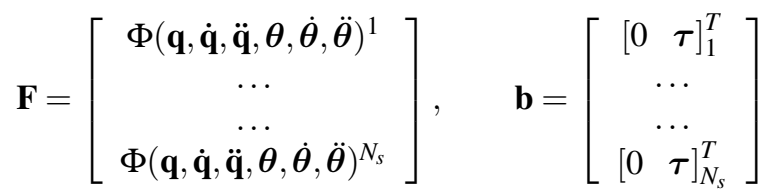

is the measured torque vector collected for $N_{s}$ samples. For the estimation here, 100 samples are collected at a frequency of $10 \mathrm{~Hz}$ over a trajectory duration of $10 \mathrm{secs}$. Due to the linear dependency of some of the columns in the $\mathbf{F}$ matrix, few parameters can only be estimated in a linear combination with other parameters, i.e., dynamic coefficients [20].

Following the parameter estimation procedure, the different models are rewritten in their respective standard regression forms, as shown in (10). This is followed by the least square estimation of each model's dynamic parameters

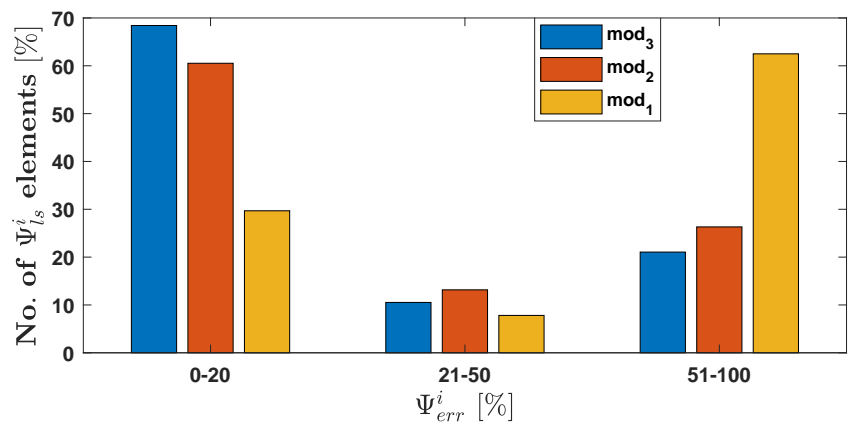

Fig. 5. The $\Psi_{\text {err }}^{i}$ is categorized into three categories $(0-20 \%, 21-50 \%$, and $51-100 \%)$ and the percentage of $\Psi_{l s}^{i}$ elements falling under each category is compared across three models: $\bmod _{1}, \bmod _{2}$, and $\bmod _{3}$.

using (11). Since the $\mathbf{F}$ matrix is likely to differ for different models, the estimated $\Psi_{l s}$ can also vary for various models. The total number of parameters that can be estimated with $\bmod _{2}$ and $\bmod _{3}$ are 75, and for $\bmod _{1}$ it drops down to 65. The parameters that cannot be estimated with $\bmod _{1}$ are the mass and inertia of cylindrical/flex spline components. This is due to the assumption of a relatively simple HD model, as discussed in Section IV-B. In the case of $\bmod _{1}$ and $\bmod _{2}$, though the actual number of parameters that can be estimated is the same, most of them are estimated in a linearly combined form, and hence, they are likely to differ.

\section{B. Comparison of Relative Estimation Error $\left(\Psi_{\text {err }}\right)$}

In order to deduce the estimation error for each model $i$, the true $\Psi^{i}$ vector is computed for each one using the actual values of the dynamic parameters, and the relative estimation error vector $\Psi_{e r r}^{i}$ in percentage is computed as shown below:

$$
\Psi_{e r r}^{i}=\left(\left(\Psi_{l s}^{i}-\Psi^{i}\right) / \Psi^{i}\right) \times 100 .
$$

Again, the individual elements of $\Psi_{\text {err }}^{i}$ of different models cannot be compared directly due to the number of parameters and its various linear combinations. Hence, the overall estimation accuracy of $\bmod _{1}, \bmod _{2}$, and $\bmod _{3}$ are compared by segregating the error into three categories: low (0-20\%), $\operatorname{mid}(21-50 \%)$, and high $(51-100 \%)$. Figure 5 compares the percentage of $\Psi_{l s}^{i}$ elements computed for each model $\bmod _{i}$ falling under three different error categories. From the figure, we can see that the relatively simple model $\bmod _{1}$ estimates $62.5 \%$ of $\Psi_{l s}^{i}$ with high error, and only $29.69 \%$ of them are estimated with low error. This trend is associated with the fact that $\bmod _{1}$ uses a relatively simple HD model and also ignores the $\mathbf{S}$ term. In the case of $\bmod _{2}$ and $\bmod _{3}$, though both the models can estimate the same number of parameters, $\bmod _{2}$ doesn't consider the $\mathbf{S}$ term. As a result, $\bmod _{3}$ reports a higher percentage $(68.42 \%)$ of $\Psi_{l s}^{i}$ estimated with low error when compared to $\bmod _{2}$ which reports a slightly lower value $(60.53 \%)$. However, with $\bmod _{2}$ and $\bmod _{3}$, a significantly higher percentage of $\Psi_{l s}^{i}$ can be estimated with a lower error when compared to $\bmod _{1}$. Also, a significantly lower percentage of $\Psi_{l s}^{i}$ have been estimated with high error using $\bmod _{2}(26.32 \%)$ and $\bmod _{3}(21 \%)$ when compared to that of $\bmod _{1}$. In the mid-range error category, the difference between 
different models seems to be minimal, with $\bmod _{2}$ reporting a slightly higher value of $13.16 \%$. Overall, $\bmod _{2}$ and $\bmod _{3}$ estimate more number of $\Psi_{l s}^{i}$ with lower error, and of which $\bmod _{3}$ being the relatively accurate model performs better across different error categories.

\section{DISCUSSION AND CONCLUSION}

1) Discussion: For this study, we haven't considered any sensor noises, and this may have some minor effects on the reported results. The noise effect will be minor because for the estimation reported in Section VI we require only joint encoders and joint torque sensors. Joint velocity and accelerations can be analytically estimated instead of offline differentiation, thanks to the use of the Fourier series wave to design the excitation trajectories. Of the two sensors, joint torque sensors are notorious for noisy measurements. Assuming a white Gaussian noise $(\mu=0, \sigma=0.26 \mathrm{Nm})$ for torque sensors [21], only the results reported for $\tau_{1}^{m}$ in Fig. $4 \mathrm{~b}$ and Fig. 4c are most likely to be dominated by noise since the maximum value is $<0.5 \mathrm{Nm}$ across different $r_{t o t}$.

In Section VI, most of the elements of $\Psi_{l s}$ are estimated in a linear combination of dynamic parameters, except for a few. Though it is possible to estimate the individual parameters from their linear combinations using non-linear optimization [20], we have chosen $\Psi_{\text {err }}$ for comparison in Section VI-B Even if the individual parameters are extracted from $\Psi_{l s}$ using [20], the overall results and observations drawn in Section VI-B may not change significantly since the extraction error is still limited by the accuracy of $\Psi_{l s}$. However, it will certainly give us more information about the exact estimation error of each parameter. Further, in Fig. 5, even for $\bmod _{2}$ and $\bmod _{3}$ around 20-30\% of $\Psi_{l s}$ have estimation error percentage $>51 \%$. This is because the elements of $\Psi_{l s}$ are in a linear combination of inertia, CoM, and mass of various components, and they are likely to result in low values due to the lower values of inertia and CoM. As a result, even a small change in $\psi_{l s}^{i}$ can result in high $\psi_{\text {err }}^{i}$ for each model as computed in (13).

2) Conclusion: In this work, the effect of ignoring $\mathbf{S}$ and adopting a simple HD model on the accuracy of a joint elastic model of an industrial manipulator has been studied. Three different models are considered here for comparison: $\bmod _{1}$, $\bmod _{2}$, and $\bmod _{3}$. The torque performance metric computed across different reduction ratios for all three models reported a relatively better accuracy of $\bmod _{3}$. Though the torque difference between the models seems to be minimal, using these models to estimate the system's dynamic parameters/coefficients resulted in a considerable error, with $\bmod _{3}$ having a relatively better estimation. The proposed detailed elastic model $\bmod _{3}$ can improve the manipulator's accuracy reported in [11], [12], [22] Experimental verification of this will be carried out in our future work.

\section{REFERENCES}

[1] H. Olsen and G. Bekey, "Identification of robot dynamics," in Proceedings. 1986 IEEE International Conference on Robotics and Automation, vol. 3. IEEE, 1986, pp. 1004-1010.
[2] P. K. Khosla and T. Kanade, "Parameter identification of robot dynamics," in 1985 24th IEEE Conference on Decision and Control. IEEE, 1985, pp. 1754-1760.

[3] M. Gautier and W. Khalil, "On the identification of the inertial parameters of robots," in Proceedings of the 27th IEEE Conference on Decision and Control, vol. 3. IEEE Austin, 1988, pp. 2264-2269.

[4] _ - "Exciting trajectories for the identification of base inertial parameters of robots," The International journal of robotics research, vol. 11, no. 4, pp. 362-375, 1992.

[5] J. Swevers, C. Ganseman, D. B. Tukel, J. De Schutter, and H. Van Brussel, "Optimal robot excitation and identification," IEEE transactions on robotics and automation, vol. 13, no. 5, pp. 730-740, 1997.

[6] J. Swevers, W. Verdonck, and J. De Schutter, "Dynamic model identification for industrial robots," IEEE control systems magazine, vol. 27 , no. 5, pp. 58-71, 2007.

[7] C. Gaz, M. Cognetti, A. Oliva, P. R. Giordano, and A. De Luca, "Dynamic identification of the franka emika panda robot with retrieval of feasible parameters using penalty-based optimization," IEEE Robotics and Automation Letters, vol. 4, no. 4, pp. 4147-4154, 2019.

[8] Z.-H. Jiang, T. Ishida, and M. Sunawada, "Neural network aided dynamic parameter identification of robot manipulators," in 2006 IEEE international conference on systems, man and cybernetics, vol. 4 IEEE, 2006, pp. 3298-3303.

[9] E. Burdet and A. Codourey, "Evaluation of parametric and nonparametric nonlinear adaptive controllers," Robotica, vol. 16, no. 1, pp. 59-73, 1998.

[10] A. Albu-Schaffer and G. Hirzinger, "Parameter identification and passivity based joint control for a 7 dof torque controlled light weight robot," in Proceedings 2001 ICRA. IEEE International Conference on Robotics and Automation (Cat. No. 01CH37164), vol. 3. IEEE, 2001, pp. 2852-2858.

[11] J. Öhr, S. Moberg, E. Wernholt, S. Hanssen, J. Pettersson, S. Persson, and S. Sander-Tavallaey, Identification of flexibility parameters of 6 axis industrial manipulator models. Linköping University Electronic Press, 2006

[12] M. Neubauer, H. Gattringer, A. Müller, A. Steinhauser, and W. Höbarth, "A two-stage calibration method for industrial robots with joint and drive flexibilities," Mechanical sciences, vol. 6, no. 2, pp. 191-201, 2015.

[13] T. Hardeman, Modelling and identification of industrial robots including drive and joint flexibilities. Universiteit Twente, 2008.

[14] B. Siciliano and O. Khatib, "Chapter 13: Robots with flexible joints," in Springer handbook of robotics. Springer, 2016, pp. 287-319.

[15] T. D. Tuttle and W. Seering, "Modeling a harmonic drive gear transmission," in [1993] Proceedings IEEE International Conference on Robotics and Automation. IEEE, 1993, pp. 624-629.

[16] W. Seyfferth, A. Maghzal, and J. Angeles, "Nonlinear modeling and parameter identification of harmonic drive robotic transmissions," in Proceedings of 1995 IEEE International Conference on Robotics and Automation, vol. 3. IEEE, 1995, pp. 3027-3032.

[17] P. Folega, G. Wojnar, R. Burdzik, and Ł. Konieczny, "Dynamic model of a harmonic drive in a toothed gear transmission system," Journal of Vibroengineering, vol. 16, no. 6, pp. 3096-3104, 2014

[18] M. W. Spong and M. Vidyasagar, "Chapter 9: Dynamics," in Robot dynamics and control. John Wiley \& Sons, 2008.

[19] L. Zollo, E. Lopez, L. Spedaliere, N. Garcia Aracil, and E. Guglielmelli, "Identification of dynamic parameters for robots with elastic joints," Advances in Mechanical Engineering, vol. 7, no. 2, p. 843186, 2015.

[20] C. Gaz, F. Flacco, and A. De Luca, "Extracting feasible robot parameters from dynamic coefficients using nonlinear optimization methods," in 2016 IEEE international conference on robotics and automation (ICRA). IEEE, 2016, pp. 2075-2081.

[21] N. Kashiri, J. Malzahn, and N. G. Tsagarakis, "On the sensor design of torque controlled actuators: A comparison study of strain gauge and encoder-based principles," IEEE Robotics and Automation Letters, vol. 2, no. 2, pp. 1186-1194, 2017.

[22] C. Gong, J. Yuan, and J. Ni, "Nongeometric error identification and compensation for robotic system by inverse calibration," International Journal of Machine Tools and Manufacture, vol. 40, no. 14, pp. 2119 2137, 2000. 